\title{
Interaction Effects of Diabetes and Hypertension on Cardio-Cerebrovascular Diseases: A Cross- Sectional Study
}

\section{Zhehui Wang}

Central South University

Tubao Yang ( $\nabla$ yangtbcsu@163.com )

Central South University https://orcid.org/0000-0001-8762-1229

Hanlin Fu

Central South University

\section{Research article}

Keywords: diabetes, hypertension, stroke, coronary heart disease

Posted Date: December 8th, 2020

DOl: https://doi.org/10.21203/rs.3.rs-122516/v1

License: (c) (i) This work is licensed under a Creative Commons Attribution 4.0 International License.

Read Full License 


\section{Abstract}

Background It is still not clear whether there exsist interaction effects of diabetes and hypertension on cardio-cerebrovascular diseases. We can not ignore it in order to prevent the incidence and progress of cardio-cerebrovascular diseases considering the high prevalence of diabetes and hypertension in the world.

Methods A cross-sectional study with a multistage stratified random sampling method was conducted among people aged 18 years or older in Changsha City, Hunan Province. A multivariate logistic regression model was performed to analyze the association between diabetes complicated with hypertension and cardio-cerebrovascular diseases. The multiplicative interaction effect was evaluated by including the product term of hypertension and diabetes in the model after adjusting for age, sex, smoking, drinking, physical exercise, and body mass index. The relative excess risk ratio (RERI), attribution percentage (AP), synergy index $(\mathrm{SI})$ and $95 \%$ confidence interval $(\mathrm{Cl})$ were calculated by the Excel calculation table compiled by Andersson to evaluate the additive interaction effects between hypertension and diabetes on cardio-cerebrovascular diseases.

Results A total of 14422 participants were recruited. The prevalence of cardio-cerebrovascular diseases in the non-diabetes and non-hypertension, diabetes, hypertension, and diabetes and hypertension combination groups was $3.7 \%, 12.1 \%, 21.2 \%$ and $31.4 \%$, respectively. The RERI, AP and SI of diabetes combined with hypertension on cardiovascular diseases were 1.92 (95\% Cl: -2.67 - 6.50), $0.23(95 \% \mathrm{Cl}$ : $-0.22-0.68)$ and 1.34 (95\% Cl: 0.69 - 2.62), respectively.

Conclusions The prevalence of cardio-cerebrovascular diseases in diabetes complicated with hypertension was higher than that in any single condition. However, no interaction effects between diabetes and hypertension were found on the prevalence of cardio-cerebrovascular diseases, indicating that the high prevalence may be due to the simple superposition of the two variables.

\section{Bankground}

At present, the main standpoint on diabetes mellitus (DM) complicated with hypertension (HT) is that their combination will increase the risk of cardio-cerebrovascular disease and death ${ }^{[1]}$. However, research on the effect of the interaction between the two variables on cardio-cerebrovascular diseases is very rare $^{[2]}$, so it is still not clear whether the increased risk caused by the combination of DM and HT could be attributed to a simple superposition or their interaction. This issue should not be ignored in studies on cardio-cerebrovascular diseases, and it is also a problem that we should consider in order to prevent the incidence and progress of cardio-cerebrovascular diseases considering the high prevalence of diabetes and hypertension in the world ${ }^{[3,4]}$.

DM and HT have been confirmed as independent risk factors for cardio-cerebrovascular diseases ${ }^{[5,6]}$, Furthermore, DM and HT are common comorbidities ${ }^{[7-9]}$. There were nearly 100 million patients with $\mathrm{HT}$, 
nearly 30 million patients with DM, and approximately 15 million patients with HT complicated with DM in China ${ }^{[10]}$. Their frequent coexistence is not a coincidence because some aspects of their pathophysiologies are shared by both conditions. $\mathrm{HT}$ is twice as frequent in patients with $\mathrm{DM}$ as in those without DM. Moreover, patients with HT often present insulin resistance and are at greater risk of developing DM than normotensive individuals. The major cause of morbidity and mortality in DM is cardio-cerebrovascular disease, which is exacerbated by $\mathrm{HT}$.

In general, physicians pay more attention to the associations between risk factors and outcomes. Thus, the interaction of risk factors on outcomes is often neglected. The term interaction refers to the situation where the effect of one risk factor on a certain disease outcome is different across the strata of another risk factor, or vice versa ${ }^{[11]}$. A biomedical study indicated that the combination of DM and HT on determinants of endothelial adhesiveness differed according to the additive effects of separate risk factors $^{[12]}$. However, even if the risk of cardio-cerebrovascular disease in DM complicated with HT was higher than that of either independent risk, few studies on the effects of the interaction between DM and HT on cardio-cerebrovascular diseases have been conducted ${ }^{[13,14]}$. A study showed that DM and HT had synergetic interactions on stroke in elderly people over 65 years old in Taiwan but only in elderly women ${ }^{[15]}$. But that study evaluated only additive interactions and not multiplicative interactions. The association between cardio-cerebrovascular diseases and DM or HT has been well documented ${ }^{[5,6]}$. However, the direction and extent to which the interactions between DM and HT affect cardiocerebrovascular diseases are unknown.

Given the high prevalence of DM and $\mathrm{HT}$, it is important to clarify the association between the combination and cardio-cerebrovascular diseases. This information can be of benefit to clinicians in the prediction, prevention, and treatment of cardio-cerebrovascular diseases. The purpose of this study focuses on elucidating whether both risk factors act synergistically to influence the outcome.

\section{Methods}

\section{Data}

Using a multistage stratified random sampling method, a community-based cross-sectional study was conducted among people aged 18 years or older chosen from each household of the included communities in 2012 in Changsha City, Hunan Province, China.

\section{Questionnaire Investigation}

A face-to-face interview was conducted by a trained team (composed of 5 investigators). The basic demographic information, lifestyle and disease status of all the participants were investigated with the unified questionnaire(supplementary 1). The basic demographic characteristics included sex, age, educational level, marital status and occupation. Lifestyle information included status of smoking, 
drinking and physical exercise, salt intake, and types of cooking oil. The disease information questionnaire included whether the following diseases were diagnosed one year ago: DM, HT, coronary heart disease(CHD) and stroke. Former and never smokers were classified as nonsmokers, and former and never alcohol drinkers were classified as nonalcohol drinkers due to the small sample sizes.

\section{Physical Examination}

The height and weight of the participants were measured by professional medical staff. Body mass index was calculated by height and weight with the following formula: body mass index = weight $(\mathrm{kg}) / \mathrm{height}$ $(\mathrm{m})^{2}$, and according to the Chinese adult obesity standard ${ }^{[16]}, \mathrm{BMI} \geqq 28.0$ was defined as obesity.

\section{Definition Of Disease}

Self-reported disease information with respect to DM, HT, CHD, and stroke was collected. In our study, HT was defined as systolic blood pressure $\geq 140 \mathrm{mmHg}$ or diastolic blood pressure $\geq 90 \mathrm{mmHg}$ at the time of the visit (mean of three readings) or a patient's self-reported history of HT or antihypertensive medications. DM was defined as fasting blood glucose $\geq 126 \mathrm{mg} / \mathrm{dL}$ or a patient's self-reported history of DM or antidiabetic medications. Cardio-cerebrovascular diseases refer to stroke, CHD, and their combination. An individual that has CHD or stroke in either or both of the conditions is considered a cardio-cerebrovascular disease patient; those who have neither of them are considered non-patients. CHD complicated with stroke refers to the condition that an individual has both CHD and stroke at the same time.

\section{Statistical analysis}

The data are presented as the mean \pm standard deviation for continuous variables and as proportions for categorical variables. Pearson's $\chi^{2}$ test was used to examine significant associations between categorical variables. Two-sample t-tests were used to examine significant associations between continuous variables. Multivariable logistic regression models were performed to identify risks for stroke, CHD and their combination. Important measures including age, sex, smoking status, alcohol consumption, physical exercise and BMI were chosen for adjustments. The relative excess risk ratio (RERI), attribution percentage (AP) and synergy index (SI) were calculated to investigate the additive interaction between DM and HT on cardio-cerebrovascular disease. An RERI score $>0$ indicated a significant synergistic interaction, and an RERI score $<0$ indicated antagonism. An AP score $>0$ indicated a significant synergistic interaction, and an AP score $<0$ indicated antagonism. An SI score $>1$ indicated a significant synergistic interaction, and an SI score $<1$ indicated antagonism. The confidence intervals (Cls) of the above indexes were estimated with the Excel calculation table compiled by Andersson ${ }^{[17]}$. $\mathrm{P}<0.05$ (twosided) was considered statistically significant. All statistical analyses were performed with SPSS for Windows 18.0 (SPSS Inc., Chicago, IL, USA) 


\section{Results}

A total of $14422($ women $=8495$, men $=5927)$ participants were recruited with a mean $(S D)$ age of 53.84 (14.88) years [P: 64.74 (12.23), NP: 52.85 (14.71)] and a mean (SD) BMI of 23.39 (3.57) kg/m2 [P: 23.89 (3.96), NP: 23.34 (3.53)]. (Table 1).

In the total population, the prevalence of cardio-cerebrovascular diseases was $8.3 \%$, and the difference in prevalence rate between participants under 60 years old (3.6\%) and over 60 years old $(16.1 \%)$ was statistically significant $(X 2=700.804, P<0.0001)$. (Table 1$)$. The prevalence of cardio-cerebrovascular diseases was $7.5 \%$ in men and $8.9 \%$ in women $\left(X^{2}=9.221, P=0.002\right), 7.5 \%$ in smokers and $8.9 \%$ in nonsmokers $\left(\chi^{2}=24.916, P<0.0001\right), 6.0 \%$ in alcohol drinkers and $9.0 \%$ in nondrinkers $\left(\chi^{2}=25.840, P<\right.$ 0.0001 ), and $11.4 \%$ in people who exercise every week and $7.7 \%$ in those who do not exercise every week $\left(X^{2}=25.840, P<0.0001\right)$. The average BMI of the respondents was $23.39 \pm 3.57 \mathrm{~kg} / \mathrm{m}^{2}$, and the patients' BMI was higher than that of the non-patients $\left(23.89 \pm 3.96\right.$ vs. $\left.23.34 \pm 3.53 \mathrm{~kg} / \mathrm{m}^{2}\right)$. The prevalence of cardio-cerebrovascular diseases was $11.3 \%$ in obese people and $8.1 \%$ in non-obese people $\left(\chi^{2}=15.409, P\right.$ $<0.0001$ ). (Table 1).

The prevalence of cardio-cerebrovascular diseases in the -DM \& -HT, +DM \& -HT, -DM \& +HT, and + DM \& $+\mathrm{HT}$ groups was $3.7 \%, 12.1 \%, 21.2 \%$, and $31.4 \%$, respectively. The differences in the prevalence rates among the four groups were statistically significant $\left(\chi^{2}=1277.008, P<0.0001\right)$. (Table 1$)$. 
Table 1

Characteristics of study participants according to prevalence of cardio-cerebrovascular diseases $(n=14422)$

\begin{tabular}{|c|c|c|c|c|}
\hline \multirow[t]{2}{*}{ Characteristics } & \multicolumn{3}{|c|}{ Prevalence of cardio-cerebrovascular diseases } & \multirow[t]{2}{*}{$P$ value } \\
\hline & Patients & Non-patients & Total & \\
\hline Age (years) & $64.74 \pm 12.23$ & $52.85 \pm 14.71$ & $53.84 \pm 14.88$ & $<0.0001$ \\
\hline$<60$ & $318(3.6 \%)$ & $8621(96.4 \%)$ & 8939 & \\
\hline$\geqq 60$ & $883(16.1 \%)$ & $4600(83.9 \%)$ & 5483 & \\
\hline Sex & & & & 0.002 \\
\hline Male & $444(7.5 \%)$ & $5483(92.5 \%)$ & 5927 & \\
\hline Female & $757(8.9 \%)$ & $7738(91.1 \%)$ & 8495 & \\
\hline Smoking status ${ }^{a}$ & & & & $<0.0001$ \\
\hline Yes & $236(6.4 \%)$ & $3460(93.6 \%)$ & 3696 & \\
\hline No & $961(9.0 \%)$ & $9693(91.0 \%)$ & 10654 & \\
\hline Alcohol consumption ${ }^{b}$ & & & & $<0.0001$ \\
\hline Yes & $173(6.0 \%)$ & $2714(94.0 \%)$ & 2887 & \\
\hline No & $1026(8.9 \%)$ & $10481(91.1 \%)$ & 11507 & \\
\hline Physical exercise ${ }^{C}$ & & & & $<0.0001$ \\
\hline Yes & $419(11.4 \%)$ & 3259 (88.6\%) & 3678 & \\
\hline No & $568(7.7 \%)$ & $6829(92.3 \%)$ & 7397 & \\
\hline BMI & $23.89 \pm 3.96$ & $23.34 \pm 3.53$ & $23.39 \pm 3.57$ & $<0.0001$ \\
\hline Obesity ${ }^{d}$ & $140(11.3 \%)$ & $1102(88.7 \%)$ & 1242 & $<0.0001$ \\
\hline Non-obesity & 1060 (8.1\%) & 12104 (91.9\%) & 13164 & \\
\hline $\mathrm{DM} \& \mathrm{H} \mathrm{T}^{\mathrm{e}}$ & & & & $<0.0001$ \\
\hline$-\mathrm{DM} \&-\mathrm{HT}$ & $399(3.7 \%)$ & $10310(96.3 \%)$ & 10709 & \\
\hline$+\mathrm{DM} \&-\mathrm{HT}$ & $55(12.1 \%)$ & $400(87.9 \%)$ & 455 & \\
\hline$-\mathrm{DM} \&+\mathrm{HT}$ & $575(21.2 \%)$ & $2135(78.8 \%)$ & 2710 & \\
\hline$+\mathrm{DM} \&+\mathrm{HT}$ & 172 (31.4\%) & $376(68.6 \%)$ & 548 & \\
\hline Total & 1201 (8.3\%) & $13221(91.7 \%)$ & 14422 & \\
\hline
\end{tabular}


Table 1. ${ }^{a}$, Former smokers and never smokers were classified as nonsmokers; ${ }^{b}$, Former drinkers and never drinkers were classified as nondrinkers; ' , People who exercise more than three times a week were considered regular exercisers; ${ }^{d}$, People with $\mathrm{BMI} \geqq 28.0$ were considered obese; ${ }^{\mathrm{e}}$, According to the status of diabetes and hypertension, the study objects can be divided into four groups: 1) without diabetes and hypertension (-DM \& -HT); 2) diabetes without hypertension (+ DM \& -HT);3) hypertension without diabetes $(-\mathrm{DM} \&+\mathrm{HT})$; and 4) diabetes with hypertension at the same time (+ DM \& $+\mathrm{HT})$. 
Table 2

Multivariate logistic regression on the risk of cardio-cerebrovascular diseases associated with DM combined with HT\# $(\mathrm{n}=14422)$

\begin{tabular}{|c|c|c|c|c|}
\hline & OR & $95 \% \mathrm{Cl}$ & Wald $\chi_{\text {multiplicative }}^{2}$ & $P_{\text {multiplicative }}$ \\
\hline \multicolumn{5}{|c|}{ Stroke or CHD } \\
\hline$-\mathrm{DM} \&-\mathrm{HT}$ & Ref. & Ref. & & \\
\hline$+\mathrm{DM} \&-\mathrm{HT}$ & 2.73 & $(1.95,3.82)$ & & \\
\hline$-\mathrm{DM} \&+\mathrm{HT}$ & 4.83 & $(4.14,5.64)$ & & \\
\hline +DM \& +HT & 8.47 & $(6.65,10.80)$ & & \\
\hline $\mathrm{DM} \times \mathrm{HT}$ & & & 4.429 & 0.035 \\
\hline \multicolumn{5}{|l|}{ Stroke } \\
\hline -DM \& -HT & Ref. & Ref. & & \\
\hline +DM \& -HT & 3.04 & $(1.35,6.86)$ & & \\
\hline$-\mathrm{DM} \&+\mathrm{HT}$ & 5.43 & $(3.66,8.06)$ & & \\
\hline +DM \& +HT & 6.34 & $(3.60,11.18)$ & & \\
\hline $\mathrm{DM} \times \mathrm{HT}$ & & & 0.327 & 0.567 \\
\hline \multicolumn{5}{|l|}{ CHD } \\
\hline -DM \& -HT & Ref. & Ref. & & \\
\hline +DM \& -HT & 2.58 & $(1.82,3.66)$ & & \\
\hline -DM \& +HT & 4.49 & $(3.83,5.27)$ & & \\
\hline +DM \& +HT & 8.13 & $(6.35,10.41)$ & & \\
\hline $\mathrm{DM} \times \mathrm{HT}$ & & & 2.713 & 0.100 \\
\hline \multicolumn{5}{|c|}{ Stroke and CHD } \\
\hline -DM \& -HT & Ref. & Ref. & & \\
\hline +DM \& -HT & 2.41 & $(0.72,8.15)$ & & \\
\hline -DM \& +HT & 4.59 & $(2.60,8.00)$ & & \\
\hline +DM \& +HT & 7.43 & $(3.58,15.41)$ & & \\
\hline $\mathrm{DM} \times \mathrm{HT}$ & & & 1.960 & 0.162 \\
\hline
\end{tabular}

Table 2. \# A multivariate logistic regression model was used to analyze the risk of cardio-cerebrovascular diseases caused by DM and HT. Five common risk factors for cardio-cerebrovascular diseases, including 
sex, age, smoking, drinking, physical exercise and BMI, were adjusted. The status of DM and HT in the study population was set as dummy variables to be included in the model, with the -DM \& -HT group as the reference group. According to the different conditions, the prevalence of cardio-cerebrovascular diseases can be divided into four categories: 1) stroke or CHD; 2) stroke; 3) CHD; and 4) stroke and CHD. The four conditions were analyzed as the dependent variables of the four regression models separately. $\mathrm{DM}, \mathrm{HT}$ and their interaction term were included in the regression model when analyzing the multiplicative effects.

Table 3

Additive interaction between DM and HT on cardio-cerebrovascular diseases ( $\mathrm{n}=$ 14422)

\begin{tabular}{|llll|}
\hline & $95 \% \mathrm{Cl}$ & & \multicolumn{1}{l|}{ SI } \\
\cline { 2 - 4 } & RERI & AP & $1.34(0.69,2.62)$ \\
\hline Stroke or CHD & $1.92(-2.67,6.50)$ & $0.23(-0.22,0.68)$ & $0.84(0.16,4.32)$ \\
\hline Stroke & $-1.01(-10.39,8.36)$ & $-0.16(-1.77,1.45)$ & $1.41(0.70,2.83)$ \\
CHD & $2.04(-2.45,6.52)$ & $0.25(-0.20,0.70)$ & $1.28(0.12,13.63)$ \\
\hline Stroke and CHD & $1.40(-12.57,15.37)$ & $0.19(-1.45,1.83)$ & 1.28 \\
\hline
\end{tabular}

The risk of cardio-cerebrovascular diseases was increased by DM combined with $\mathrm{HT}(\mathrm{OR}=8.47,95 \% \mathrm{Cl}$ : 6.65-10.80) (Table 2), adjusted for sex, age, smoking, drinking, exercise and BMI, and especially increased more for $\mathrm{CHD}(\mathrm{OR}=8.13,95 \% \mathrm{Cl}$ : $6.35-10.41)$ than for stroke $(\mathrm{OR}=6.34,95 \% \mathrm{Cl}: 3.60-11.18)$ or their combination (OR $=7.43,95 \% \mathrm{Cl}: 3.58-15.41)$. However, none of the interaction terms of DM and HT were statistically significant for cardio-cerebrovascular diseases except for their union (stroke or CHD) $(\mathrm{X} 2=4.429, \mathrm{P}=0.035)$, which indicated that $\mathrm{HT}$ and $\mathrm{DM}$ have a negative multiplicative interaction ( $\mathrm{OR}=$ $0.648,95 \%$ Cl: $0.429-0.977$ for DM $\times$ HT) on cardio-cerebrovascular diseases (stroke or CHD). (Table 2).

The results of the additive interaction suggested that DM and HT did not have addictive interactions with cardio-cerebrovascular diseases. In terms of the synergy index (SI), SI $=0.84$ for stroke $(95 \% \mathrm{Cl}$ : $0.16-$ 4.32), $\mathrm{SI}=1.41$ for $\mathrm{CHD}$ (95\% Cl: $0.70-2.83), \mathrm{SI}=1.34$ for stroke or $\mathrm{CHD}(95 \% \mathrm{Cl}: 0.69-2.62)$, and $\mathrm{SI}=1.28$ for stroke and $\mathrm{CHD}$ (95\% Cl: $0.12-13.63)$. In terms of the relative excess risk ratio (RERI), RERI $=2.45$ for CHD (95\% Cl: $-2.45-6.52)$, RERI $=1.92$ for stroke or CHD (95\% Cl: $-2.67-6.50), \mathrm{RERI}=1.40$ for stroke and CHD (95\% Cl: $-12.57-15.37)$, and RERI $=-1.01$ for stroke (95\% Cl: $-10.39-8.36)$. In terms of the attribution percentage (AP), AP $=0.25$ for $\mathrm{CHD}$ (95\% Cl: $-0.20-0.70), \mathrm{AP}=0.19$ for stroke or $\mathrm{CHD}(95 \% \mathrm{Cl}:-1.45-$ 1.83), $\mathrm{AP}=0.23$ for stroke and $\mathrm{CHD}(95 \% \mathrm{Cl}:-0.22-0.68)$, and $\mathrm{AP}=-0.16$ for stroke $(95 \% \mathrm{Cl}:-1.77-1.45)$. (Table 3).

\section{Discussion}


In general, if two factors have a positive or no multiplicative interaction, they have a positive interaction in the addition model; if two factors have a negative multiplication interaction, they may have a positive, a negative or no interaction in the addition model ${ }^{[18]}$. That is, the negative multiplicative interaction cannot prove whether the two factors have biological interactions. In this way, the results of the multiplicative interaction model and additive interaction model in our study are essentially consistent with each other in the nature of biological interactions. Namely, the combination of DM and HT can significantly increase the risk of cardio-cerebrovascular diseases compared with their existence alone, but no interaction effects were observed in our study between them on cardio-cerebrovascular diseases. The increased risk may be attributed to a simple superposition of the two.

As far as we concerned, only a few studies focused on the combined effect of DM and HT on cardiocerebrovascular diseases[2,13,14, 15, 19, 20,21]. Nevertheless, there were still 2 papers[14, 21] among this studied the independent and combined effects of the two variables but did not further study the interaction effect. They respectively are: Gang $\mathrm{Hu}^{[14]}$ found that $\mathrm{HT}$ and type $2 \mathrm{DM}$ increased the CHD risk independently, and their combination increased the risk dramatically, particularly in women. Neda Zafari ${ }^{[21]}$ found that the risk of $\mathrm{CHD}$ and stroke was different between $\mathrm{HT}$ and DM. The risk of $\mathrm{CHD}$ and stroke in DM combined with HT was higher than that in DM and higher than that in HT. The interaction effect was not analyzed in both studies. That is, only 5 papers studied the interaction effect of DM and HT on cardio-cerebrovascular diaseases $[2,13,15,19,20]$.

Yun Ju Lai ${ }^{[15]}$ conducted a cross-sectional survey of community elderly people aged 65 and above in Shipai, Taiwan. The results showed that there were sex differences in the interaction between DM and HT on stroke. DM and $\mathrm{HT}$ had synergistic additive interactions ( $\mathrm{SI}=3.16,95 \% \mathrm{Cl}: 1.35-7.39)$ but only in elderly women. However, that study had the following shortcomings: a) the study population was over 65 years old, which makes the extrapolation of conclusions limited; and b) only the additive interaction was studied, while the multiplication interaction was not explored. Although Rothman ${ }^{[22]}$ thought that the evaluation of biological interactions should be based on an additive scale rather than a multiplicative scale, there is still controversy regarding which interaction model is more in line with biological laws. And the Strengthening the Reporting of Observational Studies in Epidemiology ${ }^{[23]}$ statement suggests that both the results of additive and multiplicative interaction analyses should be reported when evaluating interactions. Most importantly, if there is truly an interaction between DM and HT on cardiocerebrovascular diseases, the interaction effects should not be limited to women but equally exist in both sexes theoretically. Therefore, the possibility of a third-order interaction on cardio-cerebrovascular diseases of DM, HT and sex cannot be excluded According his study results. Cai Huan's ${ }^{[19]}$ small sample cross-sectional study found that DM and HT had synergistic interactions on the severity of stroke (severe stroke). However, the study used patients with moderate/medium-sized stroke rather than non-patients as controls and only adjusted for age and sex, not other common cardio-cerebrovascular risk factors such as smoking, drinking, diet, physical activity, or body mass index, which may have a large residual confounding effect. Shan $\mathrm{Lu}^{[20]}$ also conducted a cross-sectional survey of 11036 people aged 15 and above in Nanjing, China. And they found that there was no multiplicative interaction between DM and HT 
on myocardial infarction and stroke. In addition, Gang Hu's further study found ${ }^{[13]}$ that the interaction terms of $\mathrm{HT}$ and DM on both stroke incidence and stroke mortality were not statistically significant, indicating that these two factors operated independently for stroke risk. However, that conclusion was also based on only the results of the multiplicative model. Zhan Yiqiang ${ }^{[2]}$ evaluated the interaction between DM and HT on cardio-cerebrovascular diseases. The results showed that HT and DM had additive interactions on cardiovascular diseases but had no interaction on stroke. That study was the most comparative with our study, but the diagnostic criteria of DM included both DM detected by blood sampling on the spot and self-reported DM of the respondents. The nonuniform standards might bring qualitative differences between on-site measurements of DM and self-reported DM; for example, selfreported diabetic patients with negative measured DM might pay more attention to health management than those with positive present DM on the spot whose status of HT and even cardio-cerebrovascular diseases might be worse than that of the former. In that way, the direction and intensity of the interaction effect might be covered due to sample selection bias. In addition, other cardio-cerebrovascular diseases other than stroke and coronary heart disease were widely included in that study, which might be responsible for the difference in results.DM combined with HT increased the risk of cardiocerebrovascular diseases, but it seems to be too early to say that DM and HT had an interactive effect on cardio-cerebrovascular diseases because no interaction effects were found in our study, whether by the multiplication model or the additive model.

Although there were no interaction effects found between DM and HT on the prevalence of cardiocerebrovascular diseases, we still need to attach great importance to them because the risk of cardiocerebrovascular diseases in DM combined with HT sharply increased almost two- to four-fold in our study when the two cardio-cerebrovascular risk factors emerged simultaneously. It was reported that DM and HT share common patient behavioral factors and pathophysiological pathways ${ }^{[24]}$. These pathways interact and influence each other and may even lead to a vicious cycle. HT and DM are components of the metabolic process of metabolic syndrome. Therefore, they may develop one after the other in the same individual ${ }^{[1]}$. Cardio-cerebrovascular disease is related to multiple risk factors. The risk of occurrence depends not only on the severity of a certain risk factor but also on the number of risk factors possessed by the individual ${ }^{[25]}$.Jonathan $\mathrm{N}^{[26]}$ found that DM and systemic hypertension(SHT) each have adverse effects on left ventricular structure and function, and the combination of DM and SHT results in the greatest degree of left ventricular hypertrophy, myocardial dysfunction, and arterial stiffness. Cesare Russo ${ }^{[27]}$ found that HT and DM are independently associated with impaired left ventricular diastolic function, independent of the effect of overweight/obesity and other covariates. Their coexistence resulted in a worse effect on left ventricular diastolic mechanics and was associated with higher left ventricular filling pressures than either condition alone. DM and $\mathrm{HT}$ are both risk factors for atherosclerosis and play roles in the formation and aggravation of endothelial and smooth muscle function ${ }^{[28]}$. In basic research, the combination of DM and HT can promote endothelial cell dysfunction ${ }^{[29]}$. The dysfunction of endothelial cells may be a change in the early stage of atherosclerosis. Both DM and HT can promote the generation of oxygen-derived free radicals, thus damaging endothelial function. When DM and HT 
coexist, endothelial cell function further decreases, and smooth muscle function is also impaired ${ }^{[28]}$. In addition, the superposition of DM and HT can promote monocyte adhesion to endothelial cells, thus increasing the production of vascular superoxide and the expression of monocyte chemoattractant protein- ${ }^{[12]}$, leading to atherosclerosis and subsequent cardio-cerebrovascular diseases. Therefore, the risk of cardio-cerebrovascular outcomes in patients with DM and HT is increased, which is biologically reasonable.

Based on the high risk of cardio-cerebrovascular diseases in patients with DM complicated with $\mathrm{HT}$, it is an urgent problem to strengthen the health management of patients with DM complicated with $\mathrm{HT}$ in order to prevent the occurrence and progression of cardio-cerebrovascular diseases ${ }^{[30]}$.

This study was a large-scale population cross-sectional study with 14422 participants. The cardiocerebrovascular diseases evaluated in our study included two major cardio-cerebrovascular diseases, namely, CHD and stroke, and their comorbidity. Both the results of the multiplicative interaction and additive interaction were reported, and the results were consistent, which provided strong support for the main conclusion; that is, DM and HT do not have interaction effects on cardio-cerebrovascular diseases. In addition, five main risk factors for cardio-cerebrovascular diseases, including sex, age, smoking, drinking and physical exercise, were adjusted, thus excluding the influence of mixed factors. However, there are still some limitations in this study: a) the information about the disease was provided by the investigators themselves, and recall bias cannot be avoided; and $b$ ) the occurrence order of $D M, H T$ and cardio-cerebrovascular diseases is unknown, so a causal relationship cannot be determined, which is a common feature of cross-sectional studies.

\section{Strengths And Limitations}

This is the first community-based cross-sectional study exploring the interaction effects of diabetes and hypertension on cardio-cerebrovascular diseases with a large-scale population of 14422 participants. Both the results of the multiplicative interaction and additive interaction were analyzed and reported, and the results were consistent, which provided strong support for the main conclusion. The multivariable logistical regression models in this study were adjusted for five recognized cardio-cerebrovascular risk factors including sex, age, smoking, drinking and physical exercise, which greatly improved the reliability of the results. This study adopted cross-sectional design, which precluded causal correlations. The information about the disease was provided by the investigators themselves, and recall bias cannot be avoided.

\section{Conclusions}

DM combined with HT significantly increased the risk of cardio-cerebrovascular diseases, but no interaction effects between them were found on cardio-cerebrovascular diseases, indicating that the high prevalence may be due to the simple superposition of the two factors. 


\section{Abbreviations}

$\mathrm{HT}$ : hypertension; DM: diabetes; CHD: coronary heart disease.

\section{Declarations}

\section{Ethics approval and consent to participate}

The study was approved by the medical ethics committee of Hunan Medical Association. Participants had provided their written informed consent to participate in this study.

\section{Consent for publication}

Not applicable this study did not involve the disclosure of personal privacy information.

\section{Availability of data and materials}

Data collected and analyzed during the current study are available from the corresponding author on reasonable request.

\section{Competing interests}

All authors declare that they have no financial relationships with any organizations that might have an interest in the submitted work and no other relationships or activities that could appear to have influenced the submitted work.

\section{Funding}

Not applicable for the current study.

\section{Authors' contributions}

Conceived the experiments, analyzed the data and wrote the paper: ZW. Performed the experiments: TY. Polished and modified the paper: HF. All authors read and approved the final manuscript.

\section{Acknowledgments}

We thank the investigators from Changsha CDC for their support.

\section{Refrence}

1. Sunkara N, H Ahsan C. Hypertension in diabetes and the risk of cardiovascular disease. CardiovasC Endocrinol. 2017;6(1):33-38.

2. Zhan YQ, Yu JM, Hu DY, Mao Y, Ding RJ, sun YH, elt. Interaction between fasting blood glucose and hypertension on cardiovascular and cerebrovascular diseases. Chinese Journal of cardiovascular 
disease. 2012;(1):57-61. Chinese.

3. Mancia G. The association of hypertension and diabetes: prevalence, cardiovascular risk and protection by blood pressure reduction. Acta Diabetol. 2005;42 Suppl 1:S17-25.

4. Xie F, Xie L, Li X, Lu LX, Ma Y, Zhan QL, elt. [Prevalence and risk factors of hypertension combined with diabetes in middle and elder population in Nan'an district of Chongqing]. Zhonghua Liu Xing Bing Xue Za Zhi. 2019;40(6):666-669. Chinese.

5. Alloubani A, Saleh A, Abdelhafiz I. Hypertension and diabetes mellitus as a predictive risk factors for stroke. Diabetes Metab Syndr. 2018;12(4):577-584.

6. Gutierrez J, Alloubani A, Mari M, Alzaatreh M. Cardiovascular Disease Risk Factors: Hypertension, Diabetes Mellitus and Obesity among Tabuk Citizens in Saudi Arabia. Open Cardiovasc Med J. 2018;12:41-49.

7. Channanath AM, Farran B, Behbehani K, Thanaraj TA. State of diabetes, hypertension, and comorbidity in Kuwait: showcasing the trends as seen in native versus expatriate populations. Diabetes Care. $2013 ; 36(6): \mathrm{e} 75$.

8. Okosun IS, Chandra KM, Choi S, Christman J, Dever GE, Prewitt TE. Hypertension and type 2 diabetes comorbidity in adults in the United States: risk of overall and regional adiposity. Obes Res. 2001;9(1):1-9.

9. Wittchen HU, Krause P, Höfler M, Pfister H, Ritz E, Göke B, elt. Diabetes mellitus und assoziierte Erkrankungen in der Allgemeinarztpraxis. Grössenordnung und Indikatoren der Belastung und der Versorgungsqualität [Hypertension, diabetes mellitus and comorbidity in primary care]. Fortschr Med Orig. 2003;121 Suppl 1:19-27. German.

10. Yu HM, Liu GZ. Relationship between hypertension, diabetes mellitus and cardiovascular disease. Molecular Cardiology of China. 2004;4(1): 52-55.

11. Ali N, Akram R, Sheikh N, Sarker AR, Sultana M. Sex-specific prevalence, inequality and associated predictors of hypertension, diabetes, and comorbidity among Bangladeshi adults: results from a nationwide cross-sectional demographic and health survey. BMJ Open. 2019;9(9):e029364.

12. Tsao PS, Niebauer J, Buitrago R, Lin PS, Wang BY, Cooke JP, elt. Interaction of diabetes and hypertension on determinants of endothelial adhesiveness. Arterioscler Thromb Vasc Biol. 1998;18(6):947-53.

13. Hu G, Sarti C, Jousilahti P, Peltonen M, Qiao Q, Antikainen R, elt. The impact of history of hypertension and type 2 diabetes at baseline on the incidence of stroke and stroke mortality. Stroke. 2005;36(12):2538-43.

14. Hu G, Jousilahti P, Tuomilehto J. Joint effects of history of hypertension at baseline and type 2 diabetes at baseline and during follow-up on the risk of coronary heart disease. Eur Heart J. 2007;28(24):3059-66.

15. Lai YJ, Chen HC, Chou P. Gender Difference in the Interaction Effects of Diabetes and Hypertension on Stroke among the Elderly in the Shih-Pai Study, Taiwan. PLoS One. 2015;10(8):e0136634. 
16. Jia W. Obesity in China: its characteristics, diagnostic criteria, and implications. Front Med. 2015;9(2):129-33.

17. Andersson T, Alfredsson L, Källberg H, Zdravkovic S, Ahlbom A. Calculating measures of biological interaction. Eur J Epidemiol. 2005;20(7):575-9.

18. KJ R modern epidemiology. Lippincott: Williams\& Wilkins press; 2008.

19. Cai H, Liu XF. Effect of interaction between diabetes mellitus and hypertension on the severity of ischemic stroke. Proceedings of the 23rd neurology conference of six provinces and one city in East China and 2016 annual meeting of Neurology of Zhejiang Province. 2016; (pp. 196-197). Ningbo, Zhejiang, China. Chinese.

20. Lu S, Bao MY, Miao SM, Zhang X, Jia QQ, Jing SQ. Prevalence of hypertension, diabetes, and dyslipidemia, and their additive effects on myocardial infarction and stroke: a cross-sectional study in Nanjing, China. Ann Transl Med. 2019;7(18):436.

21. Zafari N, Asgari S, Lotfaliany M, Hadaegh A, Azizi F, Hadaegh F. Impact Of Hypertension versus Diabetes on Cardiovascular and All-cause Mortality in Iranian Older Adults: Results of 14 Years of Follow-up. Sci Rep. 2017;7(1):14220.

22. KJ R. Epidemiology: an introduction. New York: Oxford University Press; 2002.

23. Vandenbroucke JP, von Elm E, Altman DG, Gøtzsche PC, Mulrow CD, Pocock SJ, Poole C, Schlesselman JJ, Egger M; STROBE Initiative. Strengthening the Reporting of Observational Studies in Epidemiology (STROBE): explanation and elaboration. Int J Surg. 2014;12(12):1500-24.

24. Petrie JR, Guzik TJ, Touyz RM. Diabetes, Hypertension, and Cardiovascular Disease: Clinical Insights and Vascular Mechanisms. Can J Cardiol. 2018;34(5):575-584.

25. Zhao D, Liu J, Xie W, Qi Y. Cardiovascular risk assessment: a global perspective. Nat Rev Cardiol. 2015;12(5):301-11.

26. Bella JN, Devereux RB, Roman MJ, Palmieri V, Liu JE, Paranicas M, Welty TK, Lee ET, Fabsitz RR, Howard BV. Separate and joint effects of systemic hypertension and diabetes mellitus on left ventricular structure and function in American Indians (the Strong Heart Study). Am J Cardiol. 2001;87(11):1260-5.

27. Russo C, Jin Z, Homma S, Rundek T, Elkind MS, Sacco RL, Di Tullio MR. Effect of diabetes and hypertension on left ventricular diastolic function in a high-risk population without evidence of heart disease. Eur J Heart Fail. 2010;12(5):454-61.

28. Ma L, Zhao S, Li J, Zhou Q, Gao M. Interaction of hypertension and diabetes on impairment of endothelial function. Chin Med J(Engl). 2001;114(6):563-7.

29. Widlansky ME, Gokce N, Keaney JF Jr, Vita JA. The clinical implications of endothelial dysfunction. $J$ Am Coll Cardiol. 2003;42(7):1149-60.

30. Petrie JR, Guzik TJ, Touyz RM. Diabetes, Hypertension, and Cardiovascular Disease: Clinical Insights and Vascular Mechanisms. Can J Cardiol. 2018;34(5):575-584. 


\section{Supplementary Files}

This is a list of supplementary files associated with this preprint. Click to download.

- Supplementary.docx 